\title{
Abordagens para aplicação da estratégia de postponement: estudo multicaso em empresas da indústria de alimentos
}

\author{
Approaches for implementation of the postponement strategy: \\ a multicase study in the food industry
}

\author{
Karine Araújo Ferreira ${ }^{1}$ \\ Rosane Lúcia Chicarelli Alcântara²
}

\begin{abstract}
Resumo: O propósito deste artigo foi investigar a aplicação prática do postponement em empresas alimentícias brasileiras e identificar as abordagens (passos) para aplicação desta estratégia nestas empresas. Além da maior discussão sobre o tema, a principal motivação para este trabalho é o estudo desta estratégia em um setor em que o tema é ainda pouco explorado. Foram apresentados seis estudos de caso em empresas alimentícias do segmento de fabricação de conservas de frutas, tais como processadoras de tomate e laranja. Este trabalho traz duas contribuições principais. A primeira é a apresentação de uma estrutura conceitual, com as principais abordagens para a implementação do postponement obtidas da literatura, que pode e deve ser adequada para diferentes setores da indústria. A segunda é uma primeira adequação desse quadro para empresas da indústria alimentícia, especificamente empresas processadoras de tomates e produtoras de suco de laranja. Dentre as principais conclusões deste trabalho, pode-se destacar que a aplicação do postponement é uma prática cada vez mais frequente no segmento investigado e tem transformado as relações entre empresas. Adicionalmente, foi verificado, que nenhuma das empresas estudadas apresentava uma estrutura formalizada para aplicação da estratégia de postponement, sendo sua implantação baseada apenas na experiência dos seus gestores.
\end{abstract}

Palavras-chave: Postergação. Estratégia de operações. Empresas alimentícias.

\begin{abstract}
The purpose of this article was to investigate the implementation of the postponement strategy of in Brazilian companies in the food industry and to identify approaches (steps) for implementing this strategy in these companies. In addition to the broader discussion on this topic, the main motivation for conducting this research is the study of this strategy in a sector where this topic has still been under-addressed. Six case studies were conducted in and fruit and vegetable canning companies, such as tomato and orange processing companies. This study has two major contributions: the first is to present, based on the literature, a theoretical framework for implementation of postponement, which can and should be suitable for different industry sectors. The second is the adequacy of this framework for use in food companies, especially orange juice and tomato processing companies. One of the main conclusions of this study is that postponement implementation has been increasingly used in the segment investigated, and it has been changing the relationships between companies. Additionally, it was found that none of the companies studied had a formal structure for implementing the postponement strategy, and therefore its implementation has been based only on the experience of its decision makers.
\end{abstract}

Keywords: Postponement. Operations strategy. Food industry.

\section{Introdução}

Um dos desafios das atuais cadeias de suprimento consiste em produzir exatamente o que os consumidores desejam, no local apropriado, com custo e tempo desejados pelo consumidor (WIKNER; NAIM; RUDBERG, 2007; GRAMAN, 2010). Para Van Hoek, Peelen e Comandeur (1999), uma maneira para enfrentar este desafio consiste em obter benefícios pela economia de escala para determinados estágios de conformação de produtos, deixando a etapa de diferenciação para o ponto em que a demanda é conhecida. Envolve também permitir que produtos sejam estocados em armazéns centralizados até o momento em que o pedido é realizado. Esta estratégia é conhecida como postponement e é definida por

\footnotetext{
${ }^{1}$ Departamento de Engenharia de Produção, Universidade Federal de Ouro Preto - UFOP, Rua 37, 115, CP 24, Loanda, CEP 35931-006, João Monlevade, MG, e-mail: karine@ deenp.ufop.br

${ }^{2}$ Departamento de Engenharia de Produção, Universidade Federal de São Carlos - UFSCar, Rod. Washington Luís, Km 235, CEP 13565-905, São Carlos, SP, e-mail: rosane@ dep.ufscar.br
} 
Van Hoek (2001) como uma estratégia da cadeia de suprimentos que consiste em adiar o máximo possível qualquer movimentação e/ou configuração final de produtos. Assim, o produto não é deslocado até que a localização da demanda (ou ponto de consumo) seja conhecida, ao mesmo tempo que sua configuração final (customização) só acontece quando as preferências do consumidor são conhecidas. Isto possibilita trazer o ponto no qual decisões sobre o que fazer são realizadas para mais perto do recebimento dos pedidos do consumidor, resultando em estoques e custos logísticos reduzidos (ASSUMPÇÃO; RIBEIRO, 2001; VAN HOEK, 2001; YANG; BURNS; BACKHOUSE, 2004a).

As primeiras experiências utilizando o postponement remontam à década de vinte (COUNCIL..., 1995) e, na literatura acadêmica, o termo foi introduzido primeiramente por Alderson (1950), como uma forma de ordenamento de etapas de agregação de valores nos processos de manufatura e de marketing.

Nos últimos anos, o presente tema tem despertado interesse de acadêmicos e executivos em diversos países. Trabalhos abordando o conceito postponement podem ser encontrados em diferentes áreas como logística, produção, marketing e, mais recentemente, na cadeia de suprimentos, conforme revisão bibliográfica realizada por Yang, Burns e Backhouse (2004a). Apesar da atenção crescente ao tema, Yang e Burns (2003) destacam que muito se tem escrito na literatura sobre os benefícios do postponement e a relevância de vários fatores que favorecem sua aplicação. Porém, poucos trabalhos discutem abordagens ou passos para sua implantação e pouco se conhece sobre as melhores maneiras de se implantar essa estratégia. Adicionalmente, cabe ressaltar que, embora alguns autores tenham estudado a aplicação do postponement em setores em que o conceito é extensivamente aplicado, como roupas, confecções, eletrônicos e automotivo, poucos estudos foram feitos sobre a viabilidade e o uso da teoria do postponement no setor alimentício, e o conceito ainda é pouco discutido. Neste setor, foram encontrados poucos trabalhos internacionais abordando o tema: um em empresas alimentícias da Europa; outro em uma empresa de vinho europeia e uma revisão bibliográfica (VAN HOEK, 1997, 1999; VAN DONK, 2001; WONG; POTTER; NAIM, 2011). Na indústria de alimentos brasileira, há ausência de mais pesquisas sobre o tema. Existem estudos teóricos, como o trabalho de Assumpção e Ribeiro (2001) e o estudo desenvolvido por Sampaio (2003), que, no segmento alimentício, investiga o tema em uma doceria. Porém, verifica-se a falta de pesquisas ou estudos empíricos aprofundados em empresas processadoras de alimentos.

Assim, este artigo tem o propósito de elaborar uma estrutura conceitual com as principais abordagens (passos) para implementação do postponement identificadas na literatura e confrontá-las com as abordagens obtidas com as práticas de postponement adotadas pelas empresas alimentícias, gerando assim uma estrutura conceitual adaptada para empresas deste setor.

Como a implementação prática do postponement ainda se encontra pouco discutida, principalmente na indústria de alimentos, o alcance destes objetivos são relevantes.

Este trabalho também se justifica pela importância da indústria alimentícia na atividade econômica brasileira. Segundo a ABIA (ASSOCIAÇÃO..., 2011), o setor faturou $\mathrm{R} \$ 388,7$ bilhões e criou 94 mil novos postos de trabalho em 2011, o que demonstra sua representatividade. Corrobora, ainda, o fato de ser este setor um dos principais exportadores de produtos, como é o caso do suco de laranja concentrado e congelado, um dos segmentos foco deste estudo. O Brasil é atualmente o maior produtor e exportador mundial do suco de laranja concentrado.

Adicionalmente, ao se pesquisar o setor alimentício, pode-se constatar que existem significativas diferenças neste setor em comparação a outros setores da indústria. As diferenças existentes são decorrentes das características específicas do setor alimentício, destacadas por Van Donk (2001), Van Hoek (1999) e Twede, Clarke e Tai (2000) em três dimensões: a) características do mercado; b) características do produto; e c) características do processo. Em relação ao mercado alimentício, os mesmos autores destacam que este é caracterizado por incerteza da demanda; curto tempo de entrega; grande variedade de produtos; e alta concorrência. As características do produto em uma empresa processadora de alimentos, por sua vez, podem ser distinguidas por qualidade $\mathrm{e}$ preço variáveis, devido à sazonalidade dos produtos agrícolas e o fato de estar sujeito à flutuações/ variações naturais do tempo, que influenciam não somente as preferências dos consumidores, mas também afetam o rendimento no suprimento dos produtos. Adicionalmente, matérias-primas, produtos semiacabados e produtos finais, em geral, são perecíveis e de baixo valor agregado. Por fim, em relação às características do processo, Van Hoek (1999) e Twede, Clarke e Tai (2000) afirmam que, geralmente, empresas de alimentos apresentam processos de produção contínuos e não modulares, o que oferece um desafio especial aplicar o postponement em alguns segmentos da indústria de alimento.

O método de pesquisa adotado foi o estudo de caso, realizado em seis empresas alimentícias do segmento de fabricação de conservas de frutas, particularmente empresas processadoras de suco de laranja concentrado e congelado e empresas produtoras de derivados de tomate.

Este artigo está estruturado em seis seções, incluindo o capítulo introdutório. Na seção 2, é apresentada uma 
síntese da literatura sobre postponement. O método de pesquisa adotado é discutido na seção 3. Os principais resultados dos estudos de caso são expostos na seção 4, seguidos pela sua análise e discussão (seção 5). Por fim, são apresentadas as considerações finais do trabalho (seção 6) e, em seguida, as referências bibliográficas.

\section{Postponement}

Nesta seção, é apresentada a revisão bibliográfica sobre o tema postponement, destacando suas definições, tipos e as principais abordagens para implementação dessa estratégia. Estas abordagens identificadas na literatura foram analisadas, ordenadas e reagrupadas em uma sequência de passos que constituiu a estrutura conceitual elaborada nesta pesquisa e que é apresentada ao final desta seção.

\subsection{Definição e tipos de postponement}

O postponement é uma prática que está crescendo e sendo cada vez mais difundida na literatura acadêmica e em aplicações práticas. Inicialmente, o conceito foi proposto por Alderson (1950) como uma maneira de mudar a forma, identidade ou lugar de produtos em um momento tão tarde quanto possível dentro dos processos de manufatura e distribuição física. Assim, postergar a movimentação do produto foi denominado de "postponement de tempo" (time postponement), enquanto a postergação na diferenciação do produto foi denominada de "postponement de forma" (form postponement).

Em 1965, Bucklin agregou mais detalhes ao trabalho de Alderson, estudou limites de aplicação da estratégia e criou o conceito oposto ao de postponement: o Princípio da Especulação (Principle of Speculation). Esse princípio é o inverso do postponement e consiste em finalizar as operações o mais cedo possível no processo de manufatura (BUCKLIN, 1965).

Depois de 1965, poucos trabalhos abordaram o assunto, e o tema foi retomado no final da década de 1980 por Zinn e Bowersox (1988), que propuseram que o postponement poderia ser separado em cinco diferentes tipos, quatro relacionados com alterações de forma do produto (etiquetagem, embalagem, montagem e manufatura) e o quinto relacionado ao tempo (centralização dos estoques).

Para Bowersox e Closs (1996) existem dois tipos de postponement: o postponement de manufatura (ou forma) e o postponement logístico (de tempo). $\mathrm{O}$ postponement de manufatura consiste em fabricar um produto base ou padrão em quantidades suficientes para realizar economia de escala, enquanto as características de finalização são adiadas até que os pedidos dos consumidores sejam recebidos. Já o postponement logístico consiste em manter toda a linha de produtos já acabados em estoque centralizado. $\mathrm{O}$ deslocamento dos estoques é adiado até o recebimento do pedido dos clientes. Quando a demanda ocorre, os pedidos são transportados diretamente ao varejo ou ao consumidor.

Van Hoek (1999) conceitua postponement como uma maneira para atrasar ou postergar o ponto de diferenciação do produto, isto é, atrasar os processos em que os produtos são transformados em especificações únicas para o consumidor. Este mesmo autor define também, além do postponement de forma e tempo, o postponement de lugar. Para ele, o postponement de lugar implica estocar os produtos acabados em localizações centrais, antes da demanda ocorrer (atrasando a determinação do local); enquanto o postponement de tempo consiste em atrasar a movimentação de produtos finais, efetuando-a apenas sob pedido (atrasando a determinação de tempo). Percebe-se que, para Van Hoek (1999), o postponement logístico engloba o postponement de tempo e o de lugar, ou seja, a combinação de postponement de tempo e lugar é entendida como postponement logístico.

Pagh e Cooper (1998) fizeram importante contribuição ao estabelecer nova morfologia, ao combinar os conceitos de postponement e especulação em:

- Estratégia de especulação plena: todas as operações são concretizadas o mais cedo possível na cadeia de suprimento e orientadas por previsões de demanda;

- Estratégia de postponement de manufatura: algumas operações, tais como pequenas montagens, embalagem ou fixação de rótulos, são realizadas dentro dos canais de distribuição;

- Estratégia de postponement logístico: o planejamento da produção é especulativo, porém a distribuição é adiada. Os pedidos dos clientes são atendidos a partir de estoques centralizados; e

- Estratégia de postponement pleno: representa o maior nível possível de postponement; consiste em somente realizar as operações de manufatura e logística contra o pedido do consumidor final.

Para incluir a aplicação do postponement em toda a extensão da cadeia de suprimentos, Waller, Dabholkar e Gentry (2000) consideram mais apropriada a nomenclatura dada com base nos estágios em que ele pode ocorrer, bem como seu envolvimento com os diferentes membros da cadeia de suprimentos. Assim, tais estratégias de postponement propostas pelos autores incluem o postponement upstream, postponement dowstream e postponement de distribuição. $\mathrm{O}$ postponement upstream (montante) ocorre com o retardo de ordens de materiais dos fornecedores até que se tenha informação de pedidos. É apropriado para empresas que empregam matérias-primas caras 
e para as que produzem a partir de projetos, como os construtores de reatores nucleares. Já postponement dowstream (jusante) é a postergação que agrega valor próximo da demanda e é executada pela empresa mais próxima do consumidor final (não necessariamente por quem produziu a primeira etapa). Este tipo de postponement pode ser definido como o atraso de algum tipo de mudança física no produto, após o estágio primário de manufatura, tais como manufatura adicional, adição de características, ou adição de alguma função que adicione valor para uma cadeia de suprimentos específica. Os autores mencionam também o postponement de distribuição (similar ao de lugar), que deve ocorrer depois que o produto já estiver com as configurações finais para ser consumido.

Cardoso (2002), Yang, Burns e Backhouse (2004b), Garcia-Dastugue e Lambert (2007) e outros autores de diferentes correntes literárias também propõem outras classificações de postponement, ampliando o leque de possibilidades.

O Quadro 1 sintetiza os tipos de postponement apresentados por autor.

Nesta pesquisa, adotou-se a classificação original proposta por Alderson (1950), ou seja, buscou-se investigar se as empresas analisadas adotavam o postponement de forma e/ou tempo. Além dos tipos de postponement, foram identificadas, nesta pesquisa, as atividades em que o postponement acontece: operações e fabricação, manufatura final, embalagem, etiquetagem, logística (distribuição física); bem como em qual estágio da cadeia de suprimentos o postponement acontece (montante ou jusante). Esta classificação foi adotada, pois, analisando as diferentes classificações propostas na literatura, foi possível verificar que, embora diferentes classificações e denominações de postponement tenham sido elaboradas pelos autores ao longo dos anos, grande parte delas tem o mesmo significado, sendo as classificações de postponement de tempo e de forma propostas originalmente por Alderson (1950) os principais tipos estudados.

\subsection{Aplicação do postponement em diferentes segmentos industriais}

Apesar das fundamentações teóricas sobre o tema, as empresas alimentícias têm começado a executar sistemas de produção postergada apenas recentemente.

Quadro 1. Síntese das classificações de postponement.

\begin{tabular}{|c|c|}
\hline Autor & Classificações de Postponement \\
\hline Alderson (1950) & $\begin{array}{l}\text { Postponement de forma } \\
\text { Postponement de tempo }\end{array}$ \\
\hline Zinn e Bowersox (1988) & $\begin{array}{l}\text { Postponement de forma é subdividido em } 4 \text { tipos: } \\
\text { de etiquetagem } \\
\text { de embalagem } \\
\text { de montagem } \\
\text { de fabricação } \\
\text { Postponement de tempo }\end{array}$ \\
\hline Bowersox e Closs (1996) & $\begin{array}{l}\text { Postponement de manufatura (= de forma) } \\
\text { Postponement logístico (= de tempo }+ \text { de lugar })\end{array}$ \\
\hline Van Hoek (1999) & $\begin{array}{l}\text { Postponement de forma } \\
\text { Postponement de tempo } \\
\text { Postponement de lugar }\end{array}$ \\
\hline Pagh e Cooper (1998) & $\begin{array}{l}\text { Especulação total } \\
\text { Postponement de manufatura (= de forma) } \\
\text { Postponement de logística (= de tempo + de lugar) } \\
\text { Postponement total }\end{array}$ \\
\hline Waller, Dabholkar e Gentry (2000) & $\begin{array}{l}\text { Postponement upstream } \\
\text { Postponement downstream (= postponement de forma) } \\
\text { Postponement de distribuição (= postponement de lugar) }\end{array}$ \\
\hline Cardoso (2002) & $\begin{array}{l}\text { Ausência de postergação } \\
\text { Centralização de estoques (= de lugar) } \\
\text { Diferenciação do produto (= de forma) } \\
\text { Combinações }\end{array}$ \\
\hline Yang, Burns e Backhouse (2004b) & $\begin{array}{l}\text { Postponement de projeto do produto } \\
\text { Postponement de compras (= postponement upstream) } \\
\text { Postponement de produção (= de forma) } \\
\text { Postponement logístico (= de tempo + de lugar) }\end{array}$ \\
\hline Garcia-Dastugue e Lambert (2007) & $\begin{array}{l}\text { Postponement pela mudança na sequência das atividades (= de forma) } \\
\text { Postponement baseado no tempo (= de tempo) }\end{array}$ \\
\hline
\end{tabular}


Adicionalmente, poucos trabalhos investigam a adoção do conceito nas empresas alimentícias, tendo destaque os trabalhos de Van Hoek (1997, 1999), Van Donk (2000), Sampaio (2003), Assumpção e Ribeiro (2001) e Wong, Potter e Naim (2011).

Ao estudar empresas alimentícias na Europa, Van Hoek (1999) verificou em quais atividades o postponement é aplicado, comparando-as com outras indústrias (automobilística, roupas e computadores). Além das atividades periféricas postergadas (embalagem, rótulo e manuais adicionados), avaliou-se também a postergação de atividades de manufatura final (processamento final, montagem, configuração, etc.) e a postergação das atividades de produção primária (produção de materiais base, componentes, etc.). Dentre as principais conclusões deste trabalho, o autor destaca que empresas na cadeia automotiva e indústria têxtil, em particular, aplicam postponement extensivamente, adotando tanto a postergação de atividades primárias (principais), como manufatura final e atividades periféricas. Em empresas de eletrônicos, o adiamento se concentra, principalmente, em atividades de manufatura final e periféricas. Já em empresas alimentícias, a aplicação do postponement tem se concentrado principalmente em atividades periféricas, como rotulagem e embalagem de produtos no estágio final da cadeia de suprimentos. Adicionalmente, o autor destaca que o postponement de manufatura é modestamente aplicado na indústria de alimentos, apesar de exceções como vinho e produção de açúcar. Isto acontece, pois, segundo o mesmo autor, quando comparado às outras indústrias, como a automobilística, eletrônica e têxtil, parece que os custos de distribuição física são de importância maior para as empresas de alimentos. Isto pode ser explicado pela perecibilidade de produtos alimentícios, que, geralmente, requerem equipamentos de distribuição física específicos, o que aumenta os custos de distribuição. Além disso, o baixo valor dos produtos alimentícios faz com que estes sejam bem mais sensíveis aos custos de distribuição do que aos custos do estoque. Assim, as empresas de alimentos, geralmente, são mais focadas no serviço e custos de distribuição que na customização de produtos e na aplicação do postponement. Corroborando com esses resultados, Twede, Clarke e Tai (2000) destacam que o processo de rotulagem tem sido postergado em muitas empresas na indústria de enlatados durante anos. Frutas frescas e vegetais são enlatados durante a colheita e armazenados centralmente sem etiquetas em latas. Assim, o processo de adição de etiqueta e rótulo é adiado para quando as empresas clientes do alimento compram o produto enlatado.

Em outro trabalho, Van Hoek (1997) examina aplicações de manufatura postergada em um produtor de vinho europeu. Com base neste estudo, Van Hoek (1997) elaborou modelos de decisão que ajudam a determinar qual tipo de postponement deve ser adotado conforme as características do produto, processo, tecnológicas e de mercado.

Em sua pesquisa sobre aplicação do postponement na indústria alimentícia, Van Donk (2000) analisa quais produtos, famílias ou combinações de produto e mercado podem e devem ser produzidos para estoque e quais devem ser produzidos sob pedido. Adicionalmente, uma estrutura baseada no conceito de ponto de desacoplamento foi desenvolvida pelo autor, na qual as características específicas foram adaptadas às empresas processadoras de alimentos.

Outro trabalho que pode ser destacado na indústria de alimentos é o de Assumpção e Ribeiro (2001), que desenvolveram um artigo teórico discutindo a aplicação da estratégia de postponement na relação entre fabricantes de açúcar e empresas processadoras de alimentos.

Outro trabalho que investiga a implementação do postponement em cinco conceituadas empresas de diferentes setores no Brasil é o de Sampaio (2003), que analisa a aplicação do postponement em empresas dos segmentos automotivo (2 empresas), tintas, computadores e alimentos ( 1 empresa de cada setor). $\mathrm{O}$ resultado da pesquisa indica que diferentes modalidades de postponement podem ser adotadas por uma única organização. Uma das empresas automotivas investigadas, por exemplo, adota as estratégias de postponement de manufatura (atividades de montagem final e montagem final no canal de distribuição), logístico e postponement pleno para diferentes produtos. A outra empresa automotiva adota o postponement de manufatura nas atividades de projeto de engenharia e manufatura final. A empresa têxtil, por sua vez, usa o postponement pleno, e a empresa de computadores, postponement de manufatura (atividade manufatura final). Por fim, a empresa de alimentos investigada adota também o postponement de manufatura, na atividade de manufatura final de $100 \%$ de seus produtos. Esta empresa é um fabricante de bolos e confeitos. Para atender aos desejos variados de seus clientes, essa empresa mantém em todas as lojas (franquias) produtos em estado básico, ou seja, massas que podem ser utilizadas em diversos bolos, assim como coberturas e recheios. Sendo assim, cada cliente pode escolher o bolo que deseja ou a própria loja pode confeccionar bolos extras daqueles que mais foram vendidos, ganhando maior flexibilidade e agilidade. Neste caso, a empresa utiliza a estratégia de postponement de manufatura, ou seja, mantém seus produtos em estado neutro até o último momento possível dentro de sua cadeia de suprimento. As tarefas de montagem dos bolos e doces são delegadas aos funcionários do canal de distribuição, mais próximos dos clientes e, portanto, mais aptos para conhecerem aquilo que realmente os clientes desejam. Os produtos 
são formados por módulos intercambiáveis, o que permite maior customização. A empresa possui um serviço de delivery e loja virtual em que o cliente escolhe o sabor dos bolos já existentes e tamanhos diversos daqueles presentes nas lojas. Nesses casos, os produtos começam a ser produzidos somente depois dos pedidos dos clientes.

Por fim, cabe destacar Wong, Potter e Naim (2011) que, por meio de um estudo de caso, avaliam o postponement como uma opção para melhorar o desempenho da cadeia de suprimentos em um produtor de café solúvel. Baseados neste estudo, os autores verificaram que, com o atraso nos processos de rotulagem e embalagem, até que os pedidos dos consumidores sejam conhecidos, economias de custo significativas foram obtidas. Estas economias incluem a redução do estoque de ciclo, do estoque de segurança e do estoque obsoleto não vendidos a partir de eventos promocionais. Os autores destacam também neste trabalho, as implicações de custo da configuração proposta que pode anular os benefícios potenciais estimados.

\subsection{Abordagens para aplicação do postponement}

Implantar uma estratégia de postponement nem sempre é uma tarefa fácil. Segundo Yang e Burns (2003), muito se tem escrito na literatura sobre os benefícios do postponement e a relevância de vários fatores para sua aplicação. Porém, poucos trabalhos discutem uma metodologia ou passos para sua implantação e pouco se conhece sobre as melhores maneiras de se implantar essa estratégia.

De acordo com Yang, Burns e Backhouse (2004a), para aplicar o postponement deve-se, inicialmente, identificar pontos postergáveis (pontos de desacoplamento), que são pontos de separação entre o que é produzido para estoque e o que é produzido sob encomenda. Uma estrutura para identificar e localizar os pontos de desacoplamento em empresas processadoras de alimentos é apresentada por Van Donk (2001). Segundo o mesmo autor, a determinação do ponto de desacoplamento depende, em geral, de dois conjuntos de características: características do produto e do mercado e características de estoque e processo. Entre as características do produto e do mercado, pode-se citar: habilidade de entrega exigida, tempo de entrega exigido, previsibilidade da demanda, especificidade da demanda. Já entre as características de estoque e processo, destacam-se: lead times e custos de etapas no processo, controle da manufatura e compras, custos de manutenção de estoques e valor adicionado nos pontos de estoque, risco de obsolescência (VAN DONK, 2001).

Cada característica mencionada influência a localização do ponto de desacoplamento. Em particular, para as indústrias processadoras de alimentos, o autor destaca como estas características podem influenciar as possíveis localizações do ponto de desacoplamento, conforme mostra o Quadro 2. Assim, de maneira similar à estrutura apresentada por Van Donk (2001) para as empresas alimentícias, outras estruturas para localização do ponto de desacoplamento podem ser elaboradas para outros setores da indústria.

Uma vez localizados os possíveis pontos de desacoplamento, Yang, Burns e Backhouse (2004a) afirmam que as companhias têm que examinar características do produto/produção e analisar os custos e benefícios do postponement. Modelos analíticos são frequentemente poderosos e significativos para ajudar a motivar ou justificar o postponement. Adicionalmente, uma vez que possíveis pontos postergáveis tenham sido identificados, as companhias podem criar estes pontos, ou seja, criar estruturas que possibilitem separar as atividades padronizadas e realizadas sob

Quadro 2. Estrutura para localização do ponto de desacoplamento em empresas alimentícias.

\begin{tabular}{|c|c|c|}
\hline Características do mercado & $\begin{array}{c}\text { Presença/valor na indústria de } \\
\text { alimentos }\end{array}$ & Efeito no ponto de desacoplamento \\
\hline Habilidade de entrega exigida & Alta & Jusante \\
\hline Tempo de entrega exigido & Curto & Jusante \\
\hline Previsibilidade da demanda & (quase sempre) imprevisível & Montante via divisão da informação \\
\hline Especificidade da demanda & $\begin{array}{l}\text { Grande variedade de produtos finais } \\
\text { (com recipientes comuns) }\end{array}$ & Possibilidades a montante \\
\hline $\begin{array}{c}\text { Características de processo e do } \\
\text { estoque }\end{array}$ & $\begin{array}{c}\text { Presença/valor na indústria de } \\
\text { alimentos }\end{array}$ & Efeito no ponto de desacoplamento \\
\hline Lead Times e Custos & Tempos de set-up relevantes & Jusante \\
\hline Controle de manufatura e compras & (às vezes) baixo & Jusante \\
\hline $\begin{array}{l}\text { Custos de manutenção de estoques e } \\
\text { valor adicionado nos pontos de estoque }\end{array}$ & Indefinidos (não muito claros) & - \\
\hline Risco de obsolescência & Alto & Montante \\
\hline
\end{tabular}

Fonte: Adaptado de Van Donk (2001). 
previsão das atividades que serão customizadas e realizadas sob pedido. O projeto do produto modular/ padrão pode auxiliar na criação de pontos postergáveis. Além disso, para que aplicações de postponement sejam bem sucedidas, é necessário incorporar o pensamento de postponement desde o projeto do produto. Ao mesmo tempo, as empresas têm que entender como lidar com obstáculos para uma adoção afetiva do conceito. As empresas geralmente encontram dificuldade para mudar rotinas e conhecimentos que estão profundamente enraizados na organização. Consequentemente, a cultura tradicional da companhia pode deliberadamente distorcer informações para mascarar sua intenção, não somente para competidores, mas para seus próprios fornecedores e consumidores. Ferramentas modernas como internet, electronic data interchange (EDI) e check outs automatizados podem melhorar o fluxo de informação por reduzir erros na coleta de dados e mover dados mais rapidamente. Entretanto, elas não eliminam distorções ou identificam boas informações no fluxo de informação. Para melhorar o fluxo de informação e adotar as estratégias de postponement, as empresas necessitam reconhecer a necessidade para comunicação aberta e, ao mesmo tempo, atuar como parceiros na cadeia de suprimentos.

Para autores como Van Hoek (1997), Pagh e Cooper (1998) e Sampaio (2003), após análise da viabilidade do postponement, um importante passo na aplicação dessa estratégia é adotar o tipo de postponement mais adequado às características de cada empresa e produto a ser fabricado. Estes mesmos autores propõem modelos nos quais as empresas podem se basear para tomar estas decisões. Os modelos propostos por estes autores para análise do tipo de postponement mais adequado às características de cada empresa e produto a ser fabricado pode ser visualizado mais detalhadamente em Van Hoek (1997, p.72-74), Pagh e Cooper (1998, p. 24) e Sampaio (2003, p. 86-87).

Outros passos para a aplicação do postponement são destacados por Lee e Tang (1997), que propõem uma classificação de três abordagens básicas para possibilitar a postergação de forma: padronização, projeto modular e reestruturação do processo. Já para Van Hoek, Peelen e Comandeur (1999), a falta de visão da cadeia de suprimentos global é uma das principais razões que explicam porque as aplicações de postponement ainda estão no estágio inicial. Além disso, eles destacam os seguintes fatores como importantes para a implantação do postponement: reestruturar processos; ter atenção suficiente para estimar custos e benefícios associados com a implementação de estratégias de postponement; enfatizar a importância da tecnologia de informação e comunicação (TIC); e tentar combinar as vantagens de eficiência de produção em massa com a potencial responsividade para customização em massa.
Por fim, Matthews e Syed (2004) afirmam que adotar uma estratégia de postponement requer tipicamente um reprojeto nos processos de manufatura. Postponement também exige um alto grau de colaboração e visibilidade por meio da cadeia de suprimentos. Segundo os mesmos autores, a primeira etapa para aplicação dessa estratégia consiste em suporte gerencial. Na etapa seguinte, deve-se determinar como melhor executar a estratégia. Um terceiro e importante passo é criar times interfuncionais para implementar a iniciativa de postponement. A equipe deve trabalhar para ajustar objetivos do grupo e objetivos de remuneração. Além disso, com representação de equipes interfuncionais, torna-se mais fácil ajustar como as mudanças em uma área da cadeia de suprimentos afetarão outra. O mapeamento do fluxo de informação entre as funções internas é também facilitado.

Baseada nas considerações apresentadas, uma síntese de possíveis abordagens que podem ser adotadas para a implementação do postponement, bem como a indicação de modelos e estruturas que podem auxiliar a implementação de algumas dessas abordagens, é apresentada no Quadro 3, a seguir.

\subsection{Construção da estrutura conceitual}

Nesta seção, será apresentada a estrutura conceitual desenvolvida nesta pesquisa, que tem como base as abordagens para implantação do postponement identificados na fundamentação teórica desenvolvida na seção 2.2. Com base no Quadro 3, as abordagens de diferentes autores foram agrupadas e ordenadas, gerando uma possível sequência de passos para aplicação do postponement proposta neste trabalho e apresentada na Figura 1. Os passos destacados com sublinhado na Figura 1 podem ser implementados com modelos e estruturas presentes na literatura, que são destacados no Quadro 3 e na explicação a seguir.

Conforme pode ser visualizado na Figura 1, o primeiro passo para aplicação do postponement consiste na identificação de pontos postergáveis, ou seja, na verificação se o processo é passível de postergação e qual o melhor local para aplicar o conceito. Nesta etapa, pode-se adotar as características para localização do ponto de desacoplamento propostas por Van Donk (2001) (Quadro 2).

Em seguida, a análise de viabilidade e do tipo de postponement mais viável às características da empresa e do mercado, bem como os produtos mais adequados para se adotar a estratégia devem ser analisados. Os modelos de decisão e análise de perfil propostas por Van Hoek (1997), Pagh e Cooper (1998) e Sampaio (2003) podem auxiliar nessa etapa. Estes modelos podem ser visualizados detalhadamente no trabalho destes autores. Definido o tipo de postponement mais adequado às características da empresa, o 
Quadro 3. Abordagens e modelos que auxiliam a implantação do postponement.

\begin{tabular}{|c|c|}
\hline Autor (es) & Abordagens para implementação do postponement \\
\hline $\begin{array}{l}\text { Yang, Burns e Backhouse } \\
\qquad(2004 \mathrm{a})\end{array}$ & $\begin{array}{l}\text { - identificação de pontos postergáveis; } \\
\text { - criação de pontos postergáveis; } \\
\text { - pensamento no postponement desde o projeto do produto; } \\
\text { - entendimento de como lidar com obstáculos à adoção da estratégia; } \\
\text { - reconhecimento das necessidades para comunicação aberta; } \\
\text { - atuação em parceria na cadeia de suprimentos. }\end{array}$ \\
\hline $\begin{array}{l}\text { Van Hoek (1997), Pagh e Cooper } \\
\text { (1998) e Sampaio (2003) }\end{array}$ & $\begin{array}{l}\text { - análise da viabilidade; } \\
\text { - adoção do tipo de postponement mais adequado às características de cada } \\
\text { produto e empresa. }\end{array}$ \\
\hline Lee e Tang (1997) & $\begin{array}{l}\text { - padronização e projeto modular; } \\
\text { • reestruturação de processos. }\end{array}$ \\
\hline $\begin{array}{l}\text { Van Hoek, Peelen e } \\
\text { Comandeur (1999) }\end{array}$ & $\begin{array}{l}\text { - reestruturação de processos; } \\
\text { - estimativa de custos e benefícios; } \\
\text { - ênfase na importância da tecnologia de informação; } \\
\text { - combinação de eficiência e responsividade. }\end{array}$ \\
\hline Matthews e Syed (2004) & $\begin{array}{l}\text { - suporte gerencial; } \\
\text { - determinação da melhor maneira de executar a estratégia (equipe interna ou } \\
\text { terceirização); } \\
\text { - criação de times interfuncionais para implementação da estratégia; } \\
\text { - mapeamento do fluxo de informação. }\end{array}$ \\
\hline Autor (es) & Modelos que auxiliam na aplicação de algumas abordagens \\
\hline Van Donk (2001) & - estrutura para identificar e posicionar o ponto de desacoplamento - Quadro 2 \\
\hline $\begin{array}{c}\text { Van Hoek (1997), Pagh e } \\
\text { Cooper (1998) e Sampaio (2003) }\end{array}$ & $\begin{array}{l}\text { - modelos para análise de viabilidade e escolha do tipo de postponement - Van } \\
\text { Hoek (1997, p.72-74), Pagh e Cooper (1998, p.24) e Sampaio (2003, p.86-87). }\end{array}$ \\
\hline
\end{tabular}

custo-benefício da estratégia deve ser avaliado, passando-se assim, ao entendimento de como lidar com os obstáculos e a criação dos pontos postergáveis. A criação destes pontos permite a separação das etapas da produção em duas fases: a fase empurrada e feita sob previsão e a fase puxada e realizada sob pedido do cliente. Adicionalmente, a identificação de qual elo na cadeia de suprimentos será o mais adequado e os responsáveis pelo postponement (equipe interna, terceiros ou outro membro da cadeia) é um passo importante para aplicação da estratégia.

Assim como em outras estratégias, o apoio da alta administração é essencial à adoção do conceito, bem como a criação de times interfuncionais e parcerias com outros membros da cadeia de suprimentos. A incorporação do conceito desde o projeto de produto e a reestruturação de processos e estratégias, como padronização, projeto modular e comonalidade, são significativas para o alcance do postponement, quando possível de serem adotadas. Em seguida, deve-se mapear o fluxo de informação e adotar tecnologias de informação e comunicação, uma vez que a informação da demanda no tempo certo é essencial para que a configuração final dos pedidos possa acontecer no momento em que os pedidos são realizados pelos clientes. Assim, o postponement pode ser alcançado por atrasar as operações de movimentação e/ou configuração dos produtos, cada um dos quais associado à atributos separados.
É importante notar que diferenças na reorganização interna e demandas externas para produtos requerem diferentes estratégias de postponement. Além disso, a empresa ao implantar o conceito pode necessitar, também, modificar sua estratégia durante o tempo e de acordo com mudanças no ambiente competitivo, estágio de ciclo de vida do produto, avanço tecnológico e assim por diante.

\section{Método de pesquisa}

A pesquisa realizada neste trabalho é de abordagem qualitativa e o método adotado foi estudo de caso. Segundo Yin (2001, p.32),

[...] um estudo de caso é uma investigação empírica que investiga um fenômeno contemporâneo dentro do contexto da vida real, especialmente quando os limites entre fenômeno e contexto não estão claramente definidos.

O foco desta pesquisa é direcionado para a situação presente, ou seja, a amplitude que as empresas alimentícias têm utilizado o postponement, para as abordagens ou passos adotados para a aplicação desse conceito.

Os estudos de caso desenvolvidos nesta pesquisa são de natureza exploratória, pois, apesar de haver mais de 60 anos de estudos acadêmicos sobre o tema, o processo de adoção da estratégia de postponement no 
ambiente de negócio brasileiro e, principalmente, nas empresas de alimentos é ainda pouco explorado. Além disso, esta pesquisa foi desenvolvida em múltiplos casos, para aumentar o grau de validade externa e também reduzir qualquer viés do pesquisador no decorrer da pesquisa.

A unidade de análise para o estudo de caso é a empresa processadora de alimentos (setor industrial) que adote um ou mais tipos de postponement identificados na literatura. Conforme já mencionado, este setor foi escolhido devido à falta de pesquisas acadêmicas que abordem a aplicação do postponement nele. Foram então selecionadas para esta pesquisa seis empresas processadoras de alimentos, sendo três inseridas na subclasse denominada "fabricação de sucos de frutas" e outras três, na subclasse "fabricação

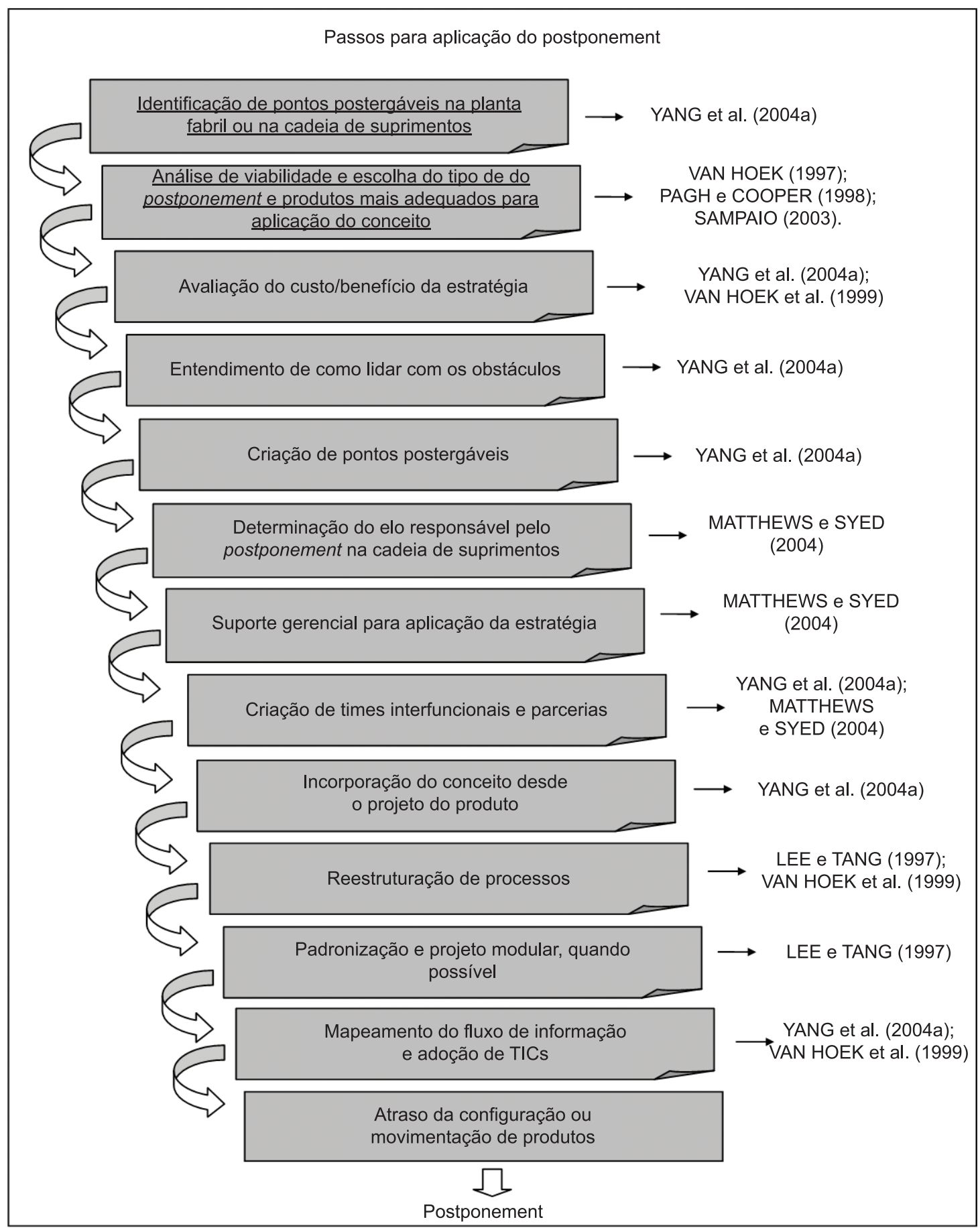

Figura 1. Passos para implantação do postponement. 
de conservas de frutas". Estas empresas foram escolhidas, pois, de acordo com a Classificação Nacional de Atividades Econômicas (2010), elas têm a maioria de seus produtos inseridos em uma mesma classe ou segmento denominado "fabricação de conservas de frutas, legumes e outros vegetais". Assim, essas empresas foram escolhidas como objeto de estudo por atender aos seguintes requisitos da pesquisa: a) serem empresas processadoras de alimentos; b) adotarem algum tipo de postponement; c) estarem disponíveis para pesquisa; d) possuírem características comuns para efeito de comparação; e) serem representativas dentro deste segmento.

Esta pesquisa foi conduzida por entrevistas semiestruturadas, realizadas com os executivos responsáveis pelas áreas da produção ou logística, permitindo confrontação das respostas para maior consistência na análise. Foram utilizadas também, nesta pesquisa, fontes de dados adicionais como relatórios contendo informações sobre aplicação do postponement e observação direta, que foi realizada de maneira informal nas visitas realizadas nas empresas para coleta de dados.

Com base na estrutura conceitual contendo as abordagens para aplicação do postponement identificados da literatura (Figura 1), foi solicitado aos entrevistados que indicassem se alguma das abordagens ou passos identificados na teoria se aplicavam nas suas empresas e se havia outros. O material coletado (nas entrevistas semiestruturadas, relatórios e observação direta) sob escopo das teorias, foi analisado, e os principais resultados são apresentados a seguir.

\section{Apresentação dos resultados}

A seguir, são apresentados os resultados dos estudos de caso realizados em seis empresas do segmento de fabricação de conservas de frutas. Por questão de sigilo, as três empresas processadoras de suco de laranja foram denominadas empresas $\mathrm{S} 1$, $\mathrm{S} 2$ e S3, e as empresas produtoras de derivados de tomate foram denominadas empresas $\mathrm{T} 1, \mathrm{~T} 2$ e T3, respectivamente.

\subsection{Caracterização das empresas}

As empresas S1, S2 e S3 estão entre as quatro maiores empresas processadoras de suco de laranja no Brasil e estão situadas no Estado de São Paulo. Já as empresas T1, T2 e T3 são empresas alimentícias que têm em comum a produção de derivados do tomate e estão entre as seis maiores empresas produtoras de derivados de tomate no Brasil. As empresas T1 e T2 estão localizadas no interior do Estado de São Paulo e a empresa T3, no interior de Goiás. As principais características dessas empresas foram sintetizadas e são apresentadas no Quadro 4.

\subsection{Adoção do postponement nas empresas alimentícias}

Na pesquisa de campo, foi possível verificar que ambos os tipos de postponement de tempo e forma são encontrados nas empresas de sucos e produtoras de derivados de tomate investigadas, sendo que no caso do postponement de forma, este ocorre principalmente nas atividades de manufatura final nas empresas estudadas.

No caso das empresas processadoras de suco de laranja, as etapas de extração do suco da laranja são realizadas pelas empresas processadoras (empresas $\mathrm{S} 1$, S2 e S3) para estoque e em formulação praticamente única dos dois principais tipos de suco produzidos por estas empresas: suco de laranja concentrado e

Quadro 4. Perfil das empresas de sucos e derivados de tomate.

\begin{tabular}{|c|c|c|c|c|c|c|}
\hline Características & Empresa S1 & Empresa S2 & Empresa S3 & Empresa T1 & Empresa T2 & Empresa T3 \\
\hline Principais Produtos & $\begin{array}{l}\text { suco de laranja } \\
\text { concentrado } \\
\text { e congelado } \\
\text { (SLCC); suco } \\
\text { integral }\end{array}$ & SLCC & $\begin{array}{l}\text { SLCC; suco } \\
\text { integral }\end{array}$ & $\begin{array}{l}\text { derivados de } \\
\text { tomate }\end{array}$ & $\begin{array}{c}\text { polpa e } \\
\text { derivados de } \\
\text { tomate e de } \\
\text { goiaba }\end{array}$ & $\begin{array}{l}\text { derivados } \\
\text { de tomate e } \\
\text { vegetais em } \\
\text { conserva }\end{array}$ \\
\hline Fundação & 1963 & 1991 & 1988 & 1955 & 1990 & 1955 \\
\hline Unidades no Brasil & 4 & 3 & 3 & 1 & 3 & 1 \\
\hline Unidades no exterior & 1 & 0 & 2 & 0 & 0 & 0 \\
\hline $\begin{array}{l}\text { Número de } \\
\text { funcionários }\end{array}$ & 1700 & $\begin{array}{c}1800 \\
\text { (entressafra) } \\
3000 \text { (safra) }\end{array}$ & $\begin{array}{c}400 \\
\text { (entressafra) } \\
3000 \text { (safra) }\end{array}$ & $\begin{array}{c}350 \\
\text { funcionários } \\
\text { diretos }\end{array}$ & $\begin{array}{c}960 \\
\text { funcionários } \\
\text { diretos }\end{array}$ & $\begin{array}{c}1300 \\
\text { funcionários } \\
\text { diretos }\end{array}$ \\
\hline Áreas de atuação & $\begin{array}{c}98 \% \\
\text { exportação }\end{array}$ & $\begin{array}{c}99 \% \\
\text { exportação }\end{array}$ & $\begin{array}{c}95 \% \\
\text { exportação }\end{array}$ & $\begin{array}{l}\text { todo o Brasil } \\
\text { e exportação }\end{array}$ & $\begin{array}{l}\text { todo o Brasil } \\
\text { e exportação } \\
(12 \%)\end{array}$ & $\begin{array}{c}\text { todo o Brasil } \\
\text { e exportação } \\
(8 \%)\end{array}$ \\
\hline Parcela de mercado & \multicolumn{3}{|c|}{$\begin{array}{c}\text { entre as quatro maiores processadoras de } \\
\text { suco de laranja do Brasil }\end{array}$} & \multicolumn{3}{|c|}{$\begin{array}{c}\text { entre as seis maiores processadoras de } \\
\text { derivados de tomate }\end{array}$} \\
\hline
\end{tabular}


congelado (SLCC); e suco de laranja integral (pronto para beber). O suco é então transportado até o país dos clientes, onde, após a chegada ao destino, pode então ser customizado (geralmente por uma empresa cliente, responsável pelo processamento final e distribuição final do suco). Assim, algumas atividades de manufatura final, como a mistura de diferentes tipos de sucos (também conhecida como processo de blendagem), diluição, adição de componentes responsáveis pelo sabor e aroma do suco, bem como adição de embalagem e rótulo, são postergadas para empresas engarrafadoras, geralmente localizadas no exterior, sendo realizadas somente no momento em que a demanda é mais conhecida.

Já para as empresas produtoras de derivados de tomate, a matéria-prima (tomate) é pré-processada, transformada em polpa e, permanece armazenada no estado semiacabado até que a demanda seja mais conhecida. Com base nas especificações de receita e demanda por determinado tipo de produto (purê, extrato, molho, entre outros), essa polpa é então transformada no produto final (derivados do tomate). Esse mesmo processo de postergação ocorre para os derivados da goiaba (doce de goiaba, goiabada cascão, goiabada em calda, entre outros) na empresa T2.

É importante destacar que, embora o postponement de forma seja verificado nas seis empresas investigadas, este ocorre em diferentes atividades e locais na cadeia de suprimentos das empresas investigadas. No caso das empresas de suco de laranja, as atividades de manufatura final, por exemplo: diluição, adição de componentes de sabor e aroma, além das atividades de envase, adição de embalagem e rótulo, só ocorrem a jusante da cadeia de suprimentos, sendo realizadas pelas empresas engarrafadoras, localizadas no exterior. Para as empresas de derivados de tomate, as atividades, geralmente, são postergadas nas próprias unidades fabris, ou seja, internamente nas empresas T1, T2 e T3, atrasando somente o momento em que as diferenciações ocorrem.

Em relação ao postponement de tempo, percebe-se que este ocorre para todos os tipos de produtos nas empresas investigadas, não apenas para o suco de laranja, derivados de tomate e goiaba. Assim, os diferentes tipos de produtos são mantidos nos armazéns localizados nas unidades produtivas das empresas investigadas e são deslocados para seus destinos (empresas engarrafadoras, no caso das empresas processadoras de suco de laranja; e para os distribuidores, supermercados e demais canais de distribuição, no caso, das empresas produtoras de derivados do tomate e goiaba) somente após o recebimento do pedido do cliente.

\subsection{Abordagens para aplicação do postponement em empresas alimentícias}

Para identificação das abordagens para aplicação do postponement adotadas pelas empresas alimentícias, foi solicitado aos entrevistados que indicassem se suas empresas adotaram algumas das abordagens identificadas previamente na literatura e se havia outras, conforme indicado no Quadro 5.

No Quadro 5, as abordagens destacadas em cinza são aquelas que não foram identificadas previamente na literatura e foram adicionadas após entrevista nas

Quadro 5. Passos para aplicação do postponement nas empresas de sucos e conservas de frutas.

\begin{tabular}{|l|c|c|c|c|c|c|}
\hline \multirow{2}{*}{\multicolumn{1}{|c}{ Passos para aplicação }} & \multicolumn{5}{c|}{ Empresas } \\
\cline { 2 - 7 } & S1 & S2 & S3 & T1 & T2 & T3 \\
\hline Identificação de pontos postergáveis & $\mathrm{X}$ & $\mathrm{X}$ & $\mathrm{X}$ & $\mathrm{X}$ & $\mathrm{X}$ & $\mathrm{X}$ \\
\hline Escolha do tipo de postponement & - & $\mathrm{X}$ & - & - & - & - \\
\hline Análise de viabilidade para aplicação & - & - & - & - & - & - \\
\hline Avaliação custo/benefício da estratégia & - & - & - & - & - & - \\
\hline Investimento em pesquisa & $\mathrm{X}$ & - & - & - & $\mathrm{X}$ & \\
\hline Entendimento de como lidar com os obstáculos & - & - & - & - & - & - \\
\hline Criação de pontos postergáveis & $\mathrm{X}$ & $\mathrm{X}$ & $\mathrm{X}$ & - & $\mathrm{X}$ & $\mathrm{X}$ \\
\hline Determinação do responsável pelo postponement & $\mathrm{X}$ & $\mathrm{X}$ & $\mathrm{X}$ & - & - & - \\
\hline Suporte gerencial da estratégia & $\mathrm{X}$ & $\mathrm{X}$ & $\mathrm{X}$ & - & $\mathrm{X}$ & $\mathrm{X}$ \\
\hline Criação de times interfuncionais e parcerias & $\mathrm{X}$ & $\mathrm{X}$ & $\mathrm{X}$ & - & - & $\mathrm{X}$ \\
\hline Incorporação do conceito desde o projeto do produto & - & $\mathrm{X}$ & $\mathrm{X}$ & $\mathrm{X}$ & $\mathrm{X}$ & $\mathrm{X}$ \\
\hline Reestruturação de processos & $\mathrm{X}$ & - & - & - & $\mathrm{X}$ & - \\
\hline Padronização de processos e projeto modular & $\mathrm{X}$ & - & $\mathrm{X}$ & $\mathrm{X}$ & $\mathrm{X}$ & $\mathrm{X}$ \\
\hline $\begin{array}{l}\text { Adoção e/ou desenvolvimento de modernas técnicas e tecnologias } \\
\text { aplicadas aos processos produtivos }\end{array}$ & - & - & - & - & $\mathrm{X}$ & $\mathrm{X}$ \\
\hline $\begin{array}{l}\text { Adoção e /ou desenvolvimento de modernas formas de } \\
\text { armazenagem e movimentação de produtos }\end{array}$ & $\mathrm{X}$ & $\mathrm{X}$ & $\mathrm{X}$ & $\mathrm{X}$ & $\mathrm{X}$ & $\mathrm{X}$ \\
\hline Adoção de tecnologias de informação e comunicação & $\mathrm{X}$ & $\mathrm{X}$ & $\mathrm{X}$ & - & $\mathrm{X}$ & $\mathrm{X}$ \\
\hline
\end{tabular}


empresas selecionadas nesta pesquisa. Entre estes, estão a adoção e desenvolvimento de: novas formas de armazenagem e movimentação de produtos; modernas técnicas e tecnologias aplicadas aos sistemas produtivos; bem como a importância de investimento em pesquisas.

Segundo os entrevistados, a adoção e/ou desenvolvimento de modernas formas de armazenagem, movimentação, técnicas e tecnologias aplicados aos sistemas produtivos estão entre os principais passos para as empresas que desejam aplicar o postponement no setor alimentício. No caso das empresas processadoras de suco de laranja, o uso de técnicas modernas e mais adequadas de acondicionamento, armazenagem e transporte do suco, como as câmaras frias e o transporte a granel, permitiram a armazenagem e transporte do suco em estado semiacabado, a baixas temperaturas e longas distâncias, uma vez que a venda do suco acontece majoritariamente em países no exterior. Já nas empresas de derivados de tomate, pode-se destacar o desenvolvimento de novas tecnologias e formas de acondicionamento do produto, como os bags assépticos, que permitem a armazenagem da polpa no período entressafra, garantindo as características de sabor e qualidade do produto final. Esta forma de acondicionamento da polpa de tomate permite que o produto seja armazenado em temperatura ambiente, sem a necessidade de condições especiais de temperatura e estocagem, reduzindo assim os custos e, ao mesmo tempo, aumentando o tempo de armazenagem do produto. Já em relação à importância de investimento em pesquisas, um exemplo citado foi o da empresa T2, que, em parceria com pesquisadores da Universidade de São Paulo (USP) e Universidade Estadual Paulista (UNESP), desenvolveu e aplicou o mesmo procedimento já adotado para o tomate para as atividades de extração, obtenção e armazenamento da polpa de goiaba, sendo pioneira nesse processo. Atualmente, a polpa de goiaba pode também ser armazenada de forma asséptica em temperatura ambiente.

Além destes passos, os entrevistados destacaram também algum dos passos já identificados previamente na literatura. Assim, foram também destacados os seguintes passos: identificação de pontos postergáveis (local e momento em que os processos poderiam ser desacoplados); criação dos pontos postergáveis (separação das atividades que deveriam ser padronizadas daquelas realizadas sob pedido do consumidor); escolha do tipo mais adequado de postponement (compras, manufatura, embalagem, etiquetagem, rótulo ou distribuição); determinação do responsável pelo postponement (no caso das empresas processadoras de suco de laranja realizado pelas empresas engarrafadoras, e nas empresas de derivados de tomate realizado internamente pelas próprias empresas); suporte gerencial da estratégia; criação de times interfuncionais e parcerias (principalmente no caso das empresas processadoras de suco de tomate, em que o processo final de elaboração do suco é postergado a outros membros da cadeia de suprimentos); adoção de tecnologias de informação e comunicação, incorporação do conceito desde o projeto de produto; reestruturação dos processos para empresas que adotam a estratégia após sua fundação; e padronização dos processos e atividades (principalmente aquelas envolvidas na elaboração do suco de laranja concentrado e congelado e polpa de tomate, que futuramente, serão transformados nos produtos finais).

A análise de viabilidade, avaliação custo-benefício e o entendimento de como lidar com os obstáculos não foram apontados pelos entrevistados como importantes abordagens para aplicação da estratégia, pois, segundo eles, a adoção do postponement passou a ser uma estratégia essencial para estas empresas para a disponibilidade do produto (fornecimento no período de safra e entressafra) e redução de custo. Além disso, como a estratégia já era usada pelos concorrentes, as empresas também tiveram que se adequar e adotá-la. As abordagens para aplicação do postponement identificadas nos estudos de caso (Quadro 5) foram estruturadas e ordenadas, gerando uma série de passos para aplicação da estratégia de postponement, que é apresentada na Figura 2.

Nesta figura, é possível visualizar que os passos destacados na teoria, em sua maioria, se aplicam às empresas alimentícias, exceto, a análise de viabilidade, a avaliação custo-benefício e o entendimento de como lidar com os obstáculos, conforme já destacado. As abordagens destacadas na pesquisa de campo e não presentes na literatura foram também acrescidas nesta figura.

\section{Discussão e análise dos resultados}

Os resultados obtidos permitiram verificar que ambos os tipos de postponement de forma e tempo selecionados da literatura foram verificados nas empresas alimentícias investigadas.

Porém, embora na literatura Van Hoek (1999) afirme que o postponement de forma (nas atividades de rótulo e embalagem) e o postponement de tempo são os principais tipos encontrados nas empresas alimentícias, foi possível verificar na pesquisa de campo que estes não são os únicos tipos de postponement que ocorrem neste setor. Nas empresas alimentícias investigadas nesta pesquisa, o postponement de forma na atividade de manufatura final também foi identificado como um dos mais aplicados.

Em relação ao estágio da cadeia de suprimentos em que o postponement acontece, foi possível verificar que, nas empresas do segmento investigado, este ocorre apenas na própria empresa fabril ou a jusante da cadeia de suprimentos, o que indica que geralmente 
o postponement ocorre em direção ao cliente final, ou seja, a jusante da empresa foco.

Analisando o Quadro 5, é possível notar que grande parte dos passos identificados na literatura foram também apontados pelas empresas investigadas, exceto a avaliação custo benefício e entendimento de como lidar com os obstáculos, que conforme já destacado não foram apontados pelos entrevistados, pois, segundo eles, a adoção do postponement passou a ser uma estratégia essencial para as empresas para a disponibilidade do produto (fornecimento no período de safra e entressafra) e redução de custo. Além disso, essa estratégia era também usada pelos concorrentes. Assim, as empresas se viram forçadas a adotar o postponement para se manterem competitivas e atenderem às disponibilidades dos clientes com rapidez.

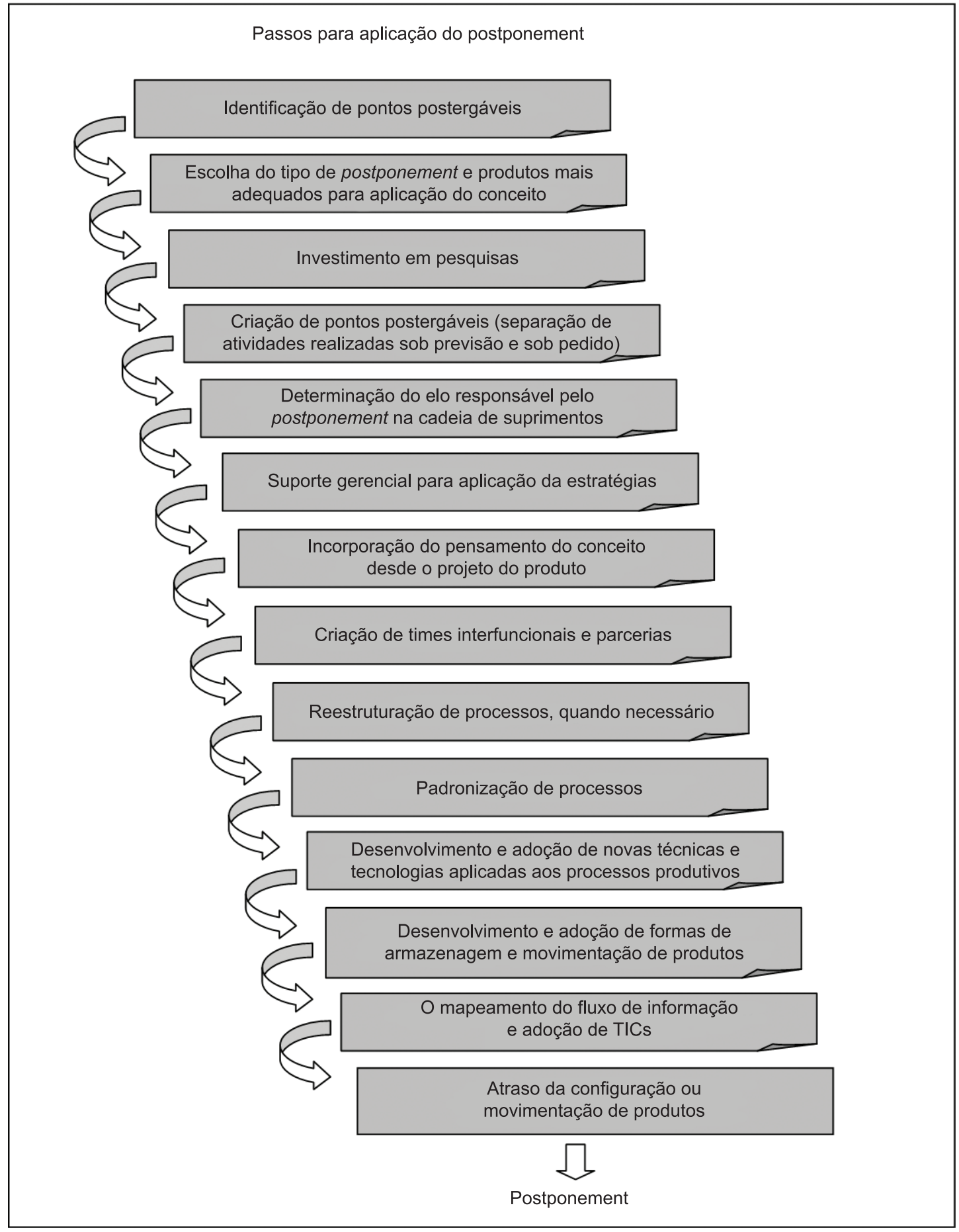

Figura 2. Passos para aplicação do postponement nas empresas alimentícias. 
Além dos passos identificados na literatura, o investimento em pesquisas e a adoção e/ou desenvolvimento de modernas técnicas e tecnologias aplicadas aos processos produtivos foram citados como importantes passos para aplicação do postponement no segmento de sucos e conservas de frutas. $\mathrm{Na}$ empresa T2, por exemplo, estes passos permitiram que o mesmo processo de postergação usado na fabricação dos derivados do tomate pudesse também ser adotado na obtenção da polpa de goiaba e posterior elaboração dos derivados da goiaba.

Outro passo não identificado na literatura e destacado pelas empresas investigadas foi a adoção e/ou desenvolvimento de modernas formas de armazenagem e movimentação de produtos. Conforme já mencionado, no caso das empresas processadoras de suco de laranja, o uso de técnicas modernas e mais adequadas de acondicionamento, armazenagem e transporte do suco, por exemplo, as câmaras frias e o transporte a granel, permitiram a armazenagem e transporte do suco em estado semiacabado, a baixas temperaturas e longas distâncias, uma vez que a venda do suco acontece majoritariamente em países no exterior. Já nas empresas de derivados de tomate, pode-se destacar o desenvolvimento de novas tecnologias e formas de acondicionamento do produto, como os bags assépticos, que permitiram a armazenagem da polpa no período entressafra em temperatura ambiente, garantindo as características de sabor e qualidade do produto final.

Percebe-se assim que, no segmento de sucos e conservas de frutas, o avanço tecnológico, principalmente no que se refere a modernas técnicas de acondicionamento e transporte dos produtos, foi condição essencial para a adoção do postponement por estas empresas, sendo o aperfeiçoamento e a adoção dessas novas técnicas um passo importante para que pudessem aplicar efetivamente essa estratégia.

As abordagens para aplicação do postponement identificadas nos estudos de caso foram estruturadas, ordenadas e apresentadas na Figura 2, gerando assim, uma estrutura conceitual para a adoção desta estratégia adaptada para empresas alimentícias, particularmente, empresas do segmento de fabricação de conservas de frutas. Esta estrutura pode servir de guia ou orientação para a adoção do postponement em empresas do setor. O objetivo não é o de fornecer uma "receita de bolo" para aplicação da estratégia de postponement, mas fornecer os resultados da pesquisa empírica que podem colaborar com as empresas na forma de uma orientação para implementação da estratégia de postponement em um setor com características específicas.

\section{Considerações finais}

Este estudo buscou aprofundar a pesquisa sobre postponement, principalmente em empresas da indústria de alimentos, setor em que existem poucas pesquisas e publicações sobre o tema. Baseado nesta investigação, o propósito deste trabalho foi investigar a implementação do postponement e identificar as abordagens (passos) para adoção desta estratégia em empresas alimentícias. Para cumprir estes objetivos foi feito um esforço de levantamento da literatura existente sobre o tema, que abrangeu literaturas da estratégia de postponement publicadas dentro dos últimos 60 anos. Adicionalmente, foi realizado um estudo multicaso em seis empresas da indústria alimentícia.

Além de maior discussão sobre o tema e a verificação de sua aplicação em empresas alimentícias, este trabalho trouxe duas contribuições principais. A primeira foi a apresentação de uma estrutura conceitual que apresenta as abordagens para implementação do postponement, que pode e deve ser adequado para diferentes setores da indústria. A segunda foi a adequação dessa estrutura (com base na pesquisa de campo) para empresas da indústria alimentícia, especificamente do segmento de fabricação de conservas de frutas, bem como a apresentação de informações que fornecem subsídios para adoção e consolidação do postponement em empresas deste setor.

É importante destacar que, apesar de poucas publicações sobre o tema no setor alimentício, a aplicação do postponement é uma prática cada vez mais frequente em empresas deste setor, particularmente no segmento de fabricação de conservas de frutas. Adicionalmente, pode-se verificar que o postponement pode oferecer mais do que condições operacionais para $\mathrm{o}$ atendimento das necessidades individuais dos consumidores com rapidez e baixo custo. A adoção desta estratégia tem também transformado as relações entre empresas. Sua implementação implica, muitas vezes, em delegar atividades de diferenciação e/ou movimentação para outros membros da cadeia de suprimentos, conforme verificado nas empresas processadoras de suco de laranja investigadas. Neste contexto, a adoção do postponement pode estreitar a relação entre os membros da cadeia de suprimentos, uma vez que exige mais coordenação e colaboração entre si.

\section{Referências}

ALDERSON, W. Marketing efficiency and the principle of postponement. Cost and Profit Outlook, n. 3, p. 15-18, 1950.

ASSOCIAÇÃO BRASILEIRA DAS INDÚSTRIAS DE ALIMENTAÇÃO - ABIA. O setor em números. São Paulo: Sala de Imprensa, 2011. Disponível em: <http:// www.abia.org.br>. Acesso em: fev. 2012.

ASSUMPÇÃO, M. R.; RIBEIRO, J. F. Compounding: postponement at processed food. In: INTERNATIONAL CONFERENCE ON AGRI-FOOD CHAIN / NETWORKS 
ECONOMICS AND MANAGEMENT, 3., 2001, Ribeirão Preto. Anais... Ribeirão Preto, 2001. 1 CD-ROM.

BOWERSOX, D. J.; CLOSS, D. J. Logistical Management: the integrated supply chain process. New York: McGraw-Hill, 1996.

BUCKLIN, L. P. Postponement, speculation and the structure of distribution channels. Journal of Marketing Research, v. 2, p. 26-31, 1965. http://dx.doi.org/10.2307/3149333

CARDOSO, P. A. O princípio da postergação: um estudo na cadeia de suprimentos das tintas para impressão. 2002. 158 f. Tese (Doutorado em Engenharia de Produção)-Universidade Catolica do Rio de Janeiro, Rio de Janeiro, 2002.

CLASSIFICAÇÃO NACIONAL DE ATIVIDADES ECONÔMICAS - CNAE. Tabela de códigos e classificações. 2010. Disponível em: <http://www. cnae.ibge.gov.br>. Acesso em: ago. 2010.

COUNCIL OF LOGISTICS MANAGEMENT. World class logistics: the challenge of managing continuous change. United State of America: Oak Book, 1995.

GARCIA-DASTUGUE, S.; LAMBERT, D. Interorganizational time-based postponement in the supply chain. Journal of Business Logistics, v. 28, n. 1, p. 57-76, 2007. http:// dx.doi.org/10.1002/j.2158-1592.2007.tb00232.x

GRAMAN, G. A. A partial-postponement decision cost model. European Journal of Operational Research, v. 201, p. 34-44, 2010. http://dx.doi.org/10.1016/j. ejor.2009.03.001

LEE, H. L.; TANG, C. S. Modelling the costs and benefits of delayed product differentiation. Management Science, v. 43, n. 1, p. 40- 53, 1997. http://dx.doi.org/10.1287/ mnsc. 43.1 .40

MATTHEWS, P.; SYED, N. The power of postponement. Supply Chain Management, Apr 2004. Disponível em: <http://www2.isye.gatech.edu/ pinar/teaching/ isye3104-fall2004/postponement.pdf>. Acesso em: fev. 2006.

PAGH, J. D.; COOPER, M. C. Postponement and speculation strategies: how to choose the right strategy. Journal of Business Logistics, v. 19, n. 2, p. 13-32, 1998.

SAMPAIO, M. O poder estratégico do postponement. 2003. 198 f.Tese (Doutorado em Administração)-Fundação Getúlio Vargas, São Paulo, 2003.

TWEDE, D.; CLARKE, R. H.; TAI, J. A. Packaging postponement: a global packaging strategy. Packaging Technology and Science, v. 13, p. 105-115, 2000. http://dx.doi.org/10.1002/10991522(200005)13:3\%3C105::AID-PTS503\%3E3.0.CO;2-9

VAN DONK, D. P. Make to stock or make to order: the decoupling point in the food processing industries. International Journal Production Economics, v. 69, p. 297-306, 2001. http://dx.doi.org/10.1016/ S0925-5273(00)00035-9
VAN HOEK, R. I. Postponed manufacturing: a case study in the food supply chain. Supply Chain Management, v. 2, n. 2, p. 18-34, 1997.

VAN HOEK, R. I. Postponement and the reconfiguration challenge for food supply chains. Supply Chain Management, v. 4, n. 1, p. 18-34, 1999. http://dx.doi. org/10.1108/13598549910255068

VAN HOEK, R. I. The rediscovery of postponement a literature review and directions for research. Journal of Operations Management, v. 19, n. 2, p. 161-184, 2001. http://dx.doi.org/10.1016/S0272-6963(00)00057-7

VAN HOEK, R. I.; PEELEN, E.; COMANDEUR, H. R. Achieving mass customization through postponement: a study of international changes. Journal of Market Focused Management, v. 3, p. 353-368, 1999. http:// dx.doi.org/10.1023/A:1009827812231

WALLER, M. A.; DABHOLKAR, P. A.; GENTRY, J. J. Postponement, product, customization, and marketoriented supply chain management. Journal of Business Logistics, v. 21, n. 2, p. 133-156, 2000.

WIKNER, J.; NAIM, M. M.; RUDBERG, M. Exploiting the Order Book for Mass Customized Manufacturing Control Systems With Capacity Limitations. IEEE Transactions on Engineering Management, v. 54, n. 1, p. 145-155, 2007. http://dx.doi.org/10.1109/ TEM.2006.889073

WONG, H.; POTTER, A.; NAIM, M. Evaluation of postponement in the soluble coffee supply chain: A case study. International Journal Production Economics, v. 131, n. 1, p. 355-364, 2011. http://dx.doi.org/10.1016/j. ijpe.2010.08.015

YANG, B.; BURNS, N. D. Implications of postponement for the supply chain. International Journal of Production Research, v. 41, n. 9, p. 2975-2090, 2003. http://dx.doi. org/10.1080/00207544031000077284

YANG, B.; BURNS, N. D.; BACKHOUSE, C. J. Postponement: review and an integrated framework. International Journal of Operations e Production Management, v. 24, n. 5, p. 268-487, 2004a. http:// dx.doi.org/10.1108/01443570410532542

YANG, B.; BURNS, N. D.; BACKHOUSE, C. J. Management of uncertainty through postponement. International Journal of Production Research, v. 42, n. 6, p. 1049-1064, 2004b. http://dx.doi.org/10.1080/0 0207540310001631601

YIN, R. K. Estudo de caso: planejamento e métodos. 2. ed. Porto Alegre: Bookman, 2001.

ZINN, W.; BOWERSOX, D.J. Planning physical distribution with the principle of postponement. Journal of Business Logistics, v. 9, n. 2, p. 117-136, 1988. 